\title{
PREVALENCIA DE NIVELES DE ANSIEDAD EN PACIENTES CON SÍNTOMAS Y SIGNOS DE DISFUNCIÓN TEMPOROMANDIBULAR
}

Prevalence of anxiety levels in patients with symptoms and signs of temporomandibular dysfunction

Fecha de Recepción: 6 de noviembre 2020
Prevalência de níveis de ansiedade em pacientes com sintomase sinais de disfunção temporomandibular

Aceptado para su publicación: 30 de noviembre 2020

\section{Autores: \\ María N Rosende $1, a$ \\ Paola B Olivera ${ }^{2, b}$ \\ Edna Y Meza $a^{2, b}$ \\ Alina N Peláez $z^{2, c}$}

1. Área Disciplinar Psicología del niño, adolescente y adulto. Facultad de Odontología. Universidad

Nacional Del Nordeste. República Argentina.

2. Área Disciplinar Metodología de la Investigación. Facultad de Odontología. Universidad Nacional Del

Nordeste. República Argentina.

a. Especialista en Docencia y Gestión Universitaria

(UNNE)

b. Odontóloga (FOUNNE)

c. Doctora de la Universidad Nacional del Nordeste en Odontología (UNNE)

Correspondencia:

Rosende, María Natalia Av. Libertad 5450, W3400 Corrientes +540379445-7992

Correo electrónico: nataliarosende@hotmail.com mnrosende@odn.unne.edu.ar

Conflicto de intereses: los autores declaran no tener conflictos de interés.

Fuente de financiamiento: Secretaría General de Ciencia y Técnica. Universidad Nacional del Nordeste.

\section{Resumen}

Evaluar la disfunción temporomandibular (DTM) contempla la inclusión de dos ejes, uno describe las características físicas y fisiológicas y otro refleja los niveles de ansiedad que originan o agravan los signos y síntomas de la disfunción. El propósito de este estudio fue determinar la prevalencia de diferentes niveles de ansiedad en pacientes que presentan síntomas y signos de disfunción temporomandibular. En un estudio observacional, descriptivo, de corte transversal, se seleccionaron 100 pacientes a partir de 18 años que concurrieron al Hospital Odontológico de la FOUNNE, según criterios de inclusión y exclusión, con previo consentimiento para la utilización de los datos. A cada paciente se le administró el Inventario de Ansiedad Rasgo-Estado (IDARE) para evaluar niveles de ansiedad y se realizó el diagnóstico clínico de signos y síntomas de DTM mediante el Test de Krogh Paulsen. Los pacientes se diferenciaron en dos grupos: DTM, con signos y síntomas de disfunción y control sin síntomas, y se categorizaron por edad en adultos jóvenes (18-35 años) y maduros (36 o más años). Se realizó un análisis descriptivo de datos empleando técnicas gráficas y un Análisis de Independencia mediante Chi-cuadrado. Se observaron similares prevalencias para los tres niveles de ansiedad rasgo, en la ansiedad estado se encontró mayor prevalencia del nivel medio. En los pacientes con signos y síntomas de DTM los niveles de ansiedad rasgo y estado fueron superiores a los pacientes del grupo control. En los dos grupos etarios, los niveles de ansiedad rasgo y estado se encuentran directamente relacionados.

Palabras clave: ansiedad, síndrome de la disfunción de articulación temporomandibular, adultos (fuente: DeCS BIREME). 


\begin{abstract}
Assessing temporomandibular dysfunction (TMD) involves the inclusion of two axes, one describing its physical and physiological characteristics and the other reflecting the levels of anxiety that cause or aggravate the signs and symptoms of the dysfunction. The purpose of this study was to determine the prevalence of different levels of anxiety in patients who present symptoms and signs of temporomandibular dysfunction. In an observational, descriptive, cross-sectional study, 100 patients over 18 years of age who attended the FOUNNE Dental Hospital were selected according to inclusion and exclusion criteria, with prior consent for the use of the data. Each patient was administered the Trait-State Anxiety Inventory (TSAI) to assess anxiety levels and the clinical diagnosis of signs and symptoms of TMD was made by means of the Krogh Paulsen Test. Patients were differentiated into two groups: TMD, with signs and symptoms of dysfunction and control without symptoms, and were categorized by age into young (18-35 years) and mature adults (36 or more years). A descriptive data analysis was performed using graphic techniques and an Independence Analysis using Chi-square. Similar prevalence was observed for the three levels of trait anxiety, in the state anxiety was found higher prevalence of the middle level. In patients with signs and symptoms of TMD, trait and status anxiety levels were higher than in the control group. In both age groups, trait and state levels of anxiety are directly related.
\end{abstract}

Key words: anxiety, temporomandibular joint dysfunction syndrome, adults (source: MeSH NLM).

\section{Resumo}

A avaliação da disfunção temporomandibular (DTM) contempla a inclusão de dois eixos, um descreve as características físicas e fisiológicas e o outro reflete os níveis de ansiedade que causam ou agravam os sinais e sintomas de disfunção. O objetivo deste estudo foi determinar a prevalência de diferentes níveis de ansiedade em pacientes com sintomas e sinais de disfunção temporomandibular. Em estudo observacional, descritivo e transversal, foram selecionados 100 pacientes com 18 anos ou mais que frequentavam o Hospital Odontológico FOUNNE, segundo critérios de inclusão e exclusão, com consentimento prévio para a utilização dos dados. O Trait-State Anxiety Inventory (IDARE) foi administrado a cada paciente para avaliar os níveis de ansiedade e o diagnóstico clínico de sinais e sintomas de DTM foi feito por meio do Teste de Krogh Paulsen. Os pacientes foram divididos em dois grupos: DTM, com sinais e sintomas de disfunção e controle sem sintomas, e foram categorizados por idade em adultos jovens (18-35 anos) e adultos (36 ou mais anos). A análise descritiva dos dados foi realizada por meio de técnicas gráficas e a Análise de Independência pelo Qui-quadrado. Prevalências semelhantes foram observadas para os três níveis de ansiedade-traço, na ansiedade-estado foi encontrada uma prevalência maior do nível médio. Em pacientes com sinais e sintomas de DTM, os níveis de ansiedade-traço e estado foram maiores do que os do grupo controle. Nas duas faixas etárias, os níveis de ansiedade-traço e estado estão diretamente relacionados.

Palavras-chave: ansiedade, síndrome da disfunção da articulação temporomandibular, adultos (fonte: DeCS BIREME).

\section{Introducción}

En el mundo actual, con vertiginosos cambios sociales, económicos, políticos y altas exigencias para la búsqueda de una mejor calidad de vida, la ansiedad se ha convertido en una causa importante de enfermedad ${ }^{1}$. Gran parte de la población se encuentra sometida constantemente a la acción de estresores y una de las respuestas del organismo a esta injuria, se refleja en el sistema estomatognático, desarrollando o acrecentando los signos y síntomas de los trastornos temporomandibulares ${ }^{2-3}$.

La articulación temporomandibular (ATM) es de vital importancia para el ser humano, interviene en disímiles funciones que tienen lugar desde momentos tan tempranos como el nacimiento del individuo, con la lactancia materna y posteriormente junto con la masticación garantiza su nutrición fisiológica. La disfunción temporomandibular (DTM) afecta a más del $50 \%$ de la población mundial en algún momento de su vida. Se plantea incluso que el $75 \%$ de la misma ha presentado alguna vez algún signo, el $33 \%$ algún síntoma y que el $5 \%$ requiere algún tipo de tratamiento ${ }^{4}$. Dentro de los factores psíquicos estudiados en relación con la DTM, uno de los más asociados es la ansiedad y sobre este tema existe bastante polémica, ya que cabe el interrogante de si es la ansiedad la causa de la disfunción o el resultado 
de tal padecimiento crónico.

Lemos et al. ${ }^{5}$, refieren que el estado psicológico del paciente se refleja en el origen multifactorial de los DTM, los autores señalan que, ante la presencia de disfunción de la ATM, se debería evaluar los trastornos emocionales o trastornos psiquiátricos del paciente para un mejor tratamiento integral y no tratar exclusivamente la ATM disfuncional. Es importante ver al ser humano desde una dimensión biopsicosocial, que contemple la multiplicidad de factores que intervienen al momento de establecer un diagnóstico preciso dado que el comportamiento de los individuos puede afectar la salud del sistema estomatognático como importante factor de riesgo ${ }^{6}$. Una de las principales características de los criterios diagnósticos modernos de DTM es la inclusión de dos ejes, uno que describe las características físicas y fisiológicas y otro que refleja los niveles de ansiedad que dan origen o agravan los signos y síntomas de esta disfunción ${ }^{6,7}$.

Krogh Paulsen, fue el pionero y líder internacional en el área de función y disfunción mandibular, sus ideas y pericia en el manejo clínico de los problemas oclusales, han tenido un importante impacto en educadores y clínicos alrededor del mundo. El índice de exploración muscular que lleva su nombre es reconocido internacionalmente ${ }^{8-10}$. Si bien en la actualidad existen muchas pruebas para el diagnóstico de los trastornos temporomandibulares, pocos han sido validados, esto es por falta de evidencia de valor diagnóstico en cuanto a sensibilidad y especificidad de estas herramientas. Esta realidad motivó a optar por tal instrumento para realizar el presente estudio y valorar el grado de sensibilidad y especificidad del Índice de exploración de Krogh Paulsen ${ }^{11}$, para conocer su potencia como herramienta diagnóstico de los problemas masticatorios de origen funcional.

Este test se compone de los items que se detallan a continuación: 1) restricción de la apertura bucal: separación entre los bordes superiores e inferiores en apertura máxima, 2) irregularidad en el movimiento de apertura y cierre: desviaciones durante los movimientos de apertura y cierre, 3 ) dolor muscular a la palpación: cuando el paciente refiriere dolor al palpar los músculos Pterigoideos externos e internos, Vientre posterior del Digástrico, Masetero y Temporal, 4) dolor en la Articulación Temporomandibular: presencia de dolor cuando el paciente abre y cierra la boca presionando el conducto auditivo externo hacia delante, 5$)$ ruidos articulares: mediante el uso de un estetoscopio, durante los movimientos de apertura y cierre, 6) traba: limitación durante la apertura o cierre, venciendo con esfuerzo, 7) posición de primer contacto distinta de posición de máxima intercuspidación: inestabilidad entre relación céntrica y posición de máxima intercuspidación, 8) posición contactante de relación céntrica: sagital de posición de máxima intercuspidación, 9) deslizamiento lateral: presencia de deslizamiento del contacto oclusal, desde relación céntrica a máxima intercuspidación.

La palabra ansiedad proviene del latín anxietas que significa congoja o aflicción, consiste en un estado de malestar psicofísico caracterizado por una turbación e inquietud y por inseguridad o temor, por lo que se vivencia como una amenaza inminente ${ }^{12}$. Por lo que resulta importante determinar cuando la ansiedad es simplemente un estado emocional y cuando es un rasgo relativamente estable de la personalidad, esto nos lleva a la distinción entre ansiedad estado y ansiedad rasgo ${ }^{13}$.

Spielberger ${ }^{14}$, considera que para definir la ansiedad de forma adecuada hay que tener en cuenta la diferenciación entre la ansiedad como estado emocional y la ansiedad como rasgo de personalidad, y por ello propuso la Teoría de Ansiedad Estado-Rasgo. La ansiedad-estado, según Spielberger, es un "estado emocional" inmediato, modificable en el tiempo, caracterizado por una combinación única de sentimientos de tensión, aprensión y nerviosismo, pensamientos molestos y preocupaciones, junto a cambios fisiológicos. Por su parte, la ansiedad-rasgo hace referencia a las diferencias individuales de ansiedad relativamente estables, siendo éstas una disposición, tendencia o rasgo. Contrariamente a la ansiedad estado, la ansiedad-rasgo no se manifiesta directamente en la conducta y debe ser inferida por la frecuencia con la que un individuo experimenta aumentos en su estado de ansiedad. En este sentido, los sujetos con alto grado de ansiedad-rasgo perciben un mayor rango de situaciones como amenazantes y están más predispuestos a sufrir ansiedad-estado de forma más frecuente o con mayor 


\section{REFO}

VOL. XIII | N² 2| AÑO 2020

ISSN 1668-7280

ISSN-E 2683-7986 intensidad $^{15-17}$.

La interacción entre ambos tipos de ansiedad explica por qué la ansiedad-estado puede variar, tanto entre individuos (la característica de ansiedad puede ser diferente ante la misma situación), como individualmente (la misma persona experimenta ansiedad en una situación, pero no en otra). En cuanto a la ansiedad-rasgo, habría que suponer que individualmente varía poco, y, debido a su influencia, las diferencias de ansiedad-estado entre individuos deberían mantenerse ampliamente estables (consistencia relativa). Finalmente, Hackfort y Spielberger ${ }^{18}$ postulan que, en caso de ansiedad-rasgo elevada, se produce un más rápido aumento de la ansiedad-estado.

Según un estudio del Observatorio Nacional De Salud Mental de Colombia realizado en el 2017, en el cual se aplicó el cuestionario de síntomas SRQ (por sus siglas en inglés: Self-Reporting Questionnaire) para adolescentes y adultos, que evalúa la presencia de síntomas de ansiedad, depresión, psicosis y epilepsia, en adultos de 18 a 44 años, el 9,6\% presenta síntomas sugestivos de algún trastorno mental, el 52,9\% tiene uno o más síntomas de ansiedad y el 80,2\% manifiesta de 1 a 3 síntomas depresivos, lo que consideran como evidencia de altos índices de ansiedad presentes en la población adulta en Colombia ${ }^{19}$.

La ansiedad es una respuesta normal del individuo ante una crisis. En la práctica clínica es necesario contar con un instrumento válido y confiable que mida los niveles de ansiedad en circunstancias específicas. El Inventario de Ansiedad Rasgo-Estado (IDARE), versión en español del STAI (State-Trait Anxiety Inventory), consta de dos escalas de autoevaluación que se utilizan para medir dos dimensiones distintas de la ansiedad ${ }^{20}$. Ansiedad-rasgo, en la que se pide a los sujetos describir cómo se sienten generalmente. Ansiedad-estado, en las que los sujetos responden cómo se sienten en un momento determinado. La ansiedad-estado es definida como "una condición o estado emocional transitorio del organismo humano, caracterizada por sentimientos de tensión y de aprehensión subjetivos conscientemente percibidos y por un aumento de la actividad del sistema nervioso autónomo". La ansiedad-rasgo "se refiere a las diferencias individuales, relativamente estables, en la propensión a la ansiedad, es decir, a las diferencias entre las personas en la tendencia a responder a situaciones percibidas como amenazantes, con elevaciones en la intensidad de la ansiedad-estado".

Numerosas investigaciones han estudiado los niveles de ansiedad mediante la prueba conocida como Inventario de Ansiedad Rasgo-Estado (IDA$\mathrm{RE})$ ideado por Spielberger en $1966^{14}$, que consiste en un inventario auto evaluativo diseñado para evaluar dos formas relativamente independientes de la ansiedad: la ansiedad como estado, comprendida como condición emocional transitoria y la ansiedad como rasgo advertida como propensión ansiosa relativamente estable.

Este inventario fue construido con el propósito de disponer de una escala, relativamente breve y confiable, para medir dos dimensiones básicas de la ansiedad. Las bases conceptuales de esta medición estuvieron en la teoría de señal de peligro de Freud, y los conceptos de Cattell acerca de las nociones de rasgo-estado. La ansiedad estado se refiere a un episodio agudo y auto limitado o a una situación provocada que no persiste más allá del evento generador, es decir, se ve solo en relación con una situación u objeto especifico, puede incidir en el curso de ciertas enfermedades precipitando el inicio de una crisis, su agravamiento y hasta su cronificación. Mientras que la ansiedad como rasgo describe un patrón longitudinal de presentación sintomática, que se ve como un estado afectivo persistente, donde la causa de la emoción es vaga o poco definida y múltiples pensamientos y eventos parecen desencadenarla ${ }^{1}$.

Este inventario también ha sido correlacionado con otras escalas en proceso de construcción para la obtención de la validez externa ${ }^{21}$. Respecto a los análisis psicométricos del inventario, las investigaciones han arrojado adecuados resultados de validez y confiabilidad en Argentina, Ecuador y Perú. Aunque el IDARE inicialmente se desarrolló para investigar fenómenos de ansiedad en adultos normales, ha sido comprobada su utilidad práctica en gran diversidad de poblaciones ${ }^{22}$.

Podemos decir que el IDARE posee una Validez 
de Contenido según el método de criterio de Jueces, confirmando así que los ítems que conforman el instrumento representan el contenido que el test objetivamente quiere valorar. En tal sentido bajo la validez de contenido el IDARE denota precisión en la definición y en grado de suficiencia.

El conocimiento de la prevalencia de signos y síntomas de DTM en relación con los diferentes niveles de ansiedad permite valorar la gravedad y origen de las manifestaciones clínicas y psicológicas y establecer indicadores de control, no solo por el grado de afectación a la salud, sino por las posibilidades que brinda para determinar una asistencia priorizada y un control real y objetivo de la terapéutica aplicada. En razón de ello este trabajo se realizó con el objetivo de determinar los diferentes niveles de ansiedad que presentan los pacientes que concurren para su atención al Hospital Universitario Odontológico de la FOUNNE con algún síntoma y signo de disfunción temporomandibular.

\section{Antecedentes}

Castillo Hernández et al. ${ }^{1}$, en el año 2001 han evaluado una muestra conformada por 100 pacientes entre los 15 y los 50 años, de ambos sexos. La mitad de ellos (grupo estudio) presentaban signos y síntomas de DTM que los llevó a acudir a consulta en busca de tratamiento, y el resto de la muestra (grupo control) no presentaba alteraciones funcionales indicadoras de DTM. Para determinar la existencia de la DTM utilizaron el test clínico de Helkimo y los niveles de ansiedad los determinaron Mediante Inventario de Ansiedad Rasgo-Estado (IDARE). Los hábitos parafuncionales que analizaron fueron: apretamiento y rechinamiento dental, mordedura de lengua, labios, carrillos y objetos, onicofagia, masticación unilateral y protracción lingual. Destacaron que el apretamiento y el rechinamiento dental fueron los síntomas más significativamente asociados a la DTM, seguidos de la masticación unilateral y la mordedura de labios, lengua y carrillos. Respecto a la ansiedad llegaron a la conclusión que entre los individuos con DTM fueron más frecuente los niveles altos de ansiedad, sobre todo de la ansiedad como rasgo de la personalidad.

Quispe Calizaya ${ }^{23}$, encontró que la prevalencia de TTM fue 98,26\%. Utilizó la prueba estadística Chi-cuadrado determinando asociación significativa entre niveles de Ansiedad-Rasgo y TTM $(p=0,003)$, no así entre niveles de Ansiedad-Estado y TTM $(p=0,14)$, concluyendo que elevados niveles de Ansiedad-Rasgo mayor es la frecuencia y severidad de TTM.

Hernández Romero et al. ${ }^{24}$, realizaron un estudio en un grupo de pacientes con TTM y encontraron que el porcentaje de pacientes con niveles de Ansiedad Estado alto era del $91 \%$ y medio del $9 \%$, lo cual indica que los trastornos en la articulación temporomandibular son altamente generadores de ansiedad y más aún, cuando existe de base esta patología como rasgo de la personalidad, donde se aprecia su manifestación en forma de alto en un $26 \%$, medio en $35 \%$ y bajo $39 \%$. El perfil de estos pacientes con TTM, está definido por tener de base niveles de ansiedad medios o altos, traducidos como trastornos de ansiedad, que pueden ser un factor desencadenante de la sintomatología dolorosa o no en la articulación temporomandibular; la que una vez instaurada, refuerza la sintomatología emocional.

Machado et al. ${ }^{25}$, han investigado la presencia de ansiedad en pacientes con DTM, atendidos en la Unidad de Investigación en Trastornos Cráneo Mandibulares de la Facultad de Odontología de la Universidad de Carabobo, durante el período julio 2007- mayo 2008. En una población constituida por 160 pacientes, seleccionaron una muestra aleatoria de 48 (30\%). Como instrumentos de recolección de datos utilizaron la Historia Clínica de Oclusión para establecer el diagnóstico de DTM y el Inventario de Ansiedad Estado-Rasgo, para evaluar dos conceptos independientes de la ansiedad: la ansiedad estado (condición emocional transitoria) y la ansiedad rasgo (propensión ansiosa relativamente estable). Demostraron que se presentó un mayor porcentaje de pacientes con DTM en el sexo femenino. En la ansiedad estado en ambos sexos, el mayor porcentaje $(56,25 \%)$ presentó un nivel normal, mientras que en la ansiedad rasgo se observó que el mayor porcentaje $(54,17 \%)$ registró un nivel bajo y un $8,3 \%$ alta ansiedad de rasgo, predominando en el sexo femenino. Como conclusiones se establecieron que, por estar estos pacientes en un estadio crónico de la DTM, la ansiedad estado se ubicó dentro de niveles norma- 
les; no pudieron afirmar que la presencia de DTM está determinada esencialmente por la existencia de una personalidad ansiosa, sino que dicha patología presenta una etiología multifactorial, donde la ansiedad rasgo puede o no jugar un papel determinante.

Luna Yañez ${ }^{26}$, en el año 2010 ha relacionado como factor psicológico el stress con la DTM en una muestra de 30 personas en edades comprendidas de 28 a 65 años, que trabajan en una empresa privada. De las 30 personas evaluadas, un $60 \%$ presentó DTM medio bajo y el complemento reportó un nivel medio alto. Al relacionar los niveles del estrés con la DTM hallaron que cuando el estrés está en niveles medio altos, la prevalencia de disfunción temporomandibular ocurrió en un $72,3 \%$, y cuando el estrés está en niveles altos la prevalencia de disfunción ocurre en un 5,6\%, el análisis estadístico, utilizando la prueba no paramétrica de Chi Cuadrado, no permitió encontrar significancia estadística para esta relación de las variables $(p>0,05)$. De las 30 personas evaluadas 18 de ellas presentaron el diagnóstico de disfunción de los cuales el 72,2 \% presentaron el diagnóstico de stress medio alto. De las 12 personas sin disfunción un 91,7 \% presentó diagnóstico de stress medio alto. Resulta de interés a partir de estos antecedentes propiciar el estudio de la relación entre los trastornos temporomandibulares y los niveles de ansiedad.

\section{Materiales y Métodos}

Este trabajo se llevó a cabo mediante un protocolo de investigación aprobado por el Comité de Bioética de la Facultad de Odontología de la Universidad Nacional del Nordeste (FOUNNE, Ord N 78-2004 - 23/4/2014).

Se realizó un estudio de tipo descriptivo observacional, de corte transversal. La población de estudio estuvo constituida por todos los pacientes que asistieron al Hospital Universitario Odontológico de la FOUNNE, entre los meses de agosto y octubre de 2016 para su atención integral, sobre los cuales se aplicaron los siguientes criterios de inclusión: edad de 18 años o más. Los criterios de exclusión fueron: 1) diagnóstico de padecimientos sistémicos que pudieran afectar al sistema estomatognático y a la ATM, antecedentes de traumatismo en el maxilar inferior o en la zona de la ATM, 2) recibir o haber recibido terapia para los trastornos de DTM cualquier tipo de tratamiento ortopédico u ortodóncico o tratamiento psicológico, 3) desdentados totales y pacientes con periodontopatías, 4) pacientes con alguna patología sistémica significativa con deficiencia motriz o neurológica, 5) en primer término, a los pacientes seleccionados se le presentó una hoja informativa sobre los procedimientos y objetivos del trabajo, los que accedieron a formar parte del estudio firmaron un consentimiento informado y pasaron a conformar la muestra, 6) la población objeto quedó integrada por 417 pacientes, entre ellos se seleccionaron 100 mediante un Muestreo al Azar Sistemático, con intervalos de 1 en 4, estableciendo una muestra del $25 \%$ de la población en estudio.

Dentro de la muestra se diferenciaron dos grupos: 1) Grupo DTM: formado por aquellos pacientes con signos y síntomas de DTM, 2) Grupo CONTROL: formado por aquellos pacientes sin signos ni síntomas de DTM.

El examen clínico de los pacientes se llevó a cabo en el Hospital Odontológico de la FOUNNE.

A todos los pacientes seleccionados, un psicólogo evaluó los niveles de ansiedad mediante la prueba conocida como Inventario de Ansiedad Rasgo-Estado (IDARE), ideado por Spielberger ${ }^{14}$.

El IDARE está formado por dos escalas de autoevaluación que recogen los valores individuales para la ansiedad estado (AE) y la ansiedad rasgo (AR). La AE describe cómo el sujeto se siente en ese momento y se traduce en niveles específicos de síntomas tales como la intranquilidad, el desasosiego y la inseguridad, todos vinculados a una situación específica mientras que la AR existe como característica, componente o atributo más o menos estable de la personalidad y constituye un rasgo propio que diferencia la actividad de la personalidad de la actividad de otros seres humanos.

Seguidamente, los pacientes fueron evaluados por un odontólogo especializado, que se encargó de realizar un diagnóstico clínico de los signos y síntomas de las DTM, mediante la prueba de Krogh Paul- 


\section{REFO}

VOL. XIII | N² 2| AÑO 2020

ISSN 1668-7280

ISSN-E 2683-7986 $\operatorname{sen}^{8}$, la cual se compone de nueve ítems, que permiten explorar de forma ordenada y secuencial los distintos componentes de la ATM (grupos musculares, articulación mandibular y oclusión dentaria).

\section{Las variables en estudio fueron:}

1) Ansiedad: como resultado de la aplicación del IDARE, los diferentes niveles de ansiedad fueron clasificados según: ansiedad rasgo (AR): (1) baja, (2) media y (3) alta; ansiedad estado (AE): (1) baja, (2) media y (3) alta.

2) Disfunción temporomandibular (DTM): como resultado de la prueba de Krogh Paulsen, los pacientes se agruparon en diferentes niveles de disfunción temporomandibular: 0 sin disfunción, 1 disfunción leve, 2 disfunción moderada y 3 disfunción severa. En el Anexo III se presenta el formulario utilizado para la aplicación del test de Krogh Paulsen.

3) Edad (ED): se registró la edad de los pacientes $\mathrm{y}$, posteriormente, se categorizó esta variable (CATED) formando dos grupos etarios que cubren el intervalo de edad de las personas que acudieron al Hospital en el período de estudio de la presente investigación: de 18 a 35 años, adultos jóvenes (1); y de 36 o más, adultos maduros (2).

4) Género (GEN): se registró el género de los pacientes (1: femenino, 2: masculino).

Los datos obtenidos fueron volcados en un archivo Excel para su posterior análisis. A los fines de visualizar el comportamiento general de las variables estudiadas, se realizó un análisis exploratorio de los datos, de manera gráfica mediante Gráficos de Caja y de Barra y analítica a través del cálculo de Estimadores o Estadísticos, que consistieron en Medidas Descriptivas para las variables cuantitativas y la construcción de Tablas de Frecuencias para las variables cualitativas. Con la finalidad de probar la hipótesis de igualdad entre los niveles de ansiedad rasgo y ansiedad estado en los grupos CONTROL y DTM se realizaron Pruebas de Wilcoxon (Mann-Whitney U). Esta prueba permite testar la hipótesis que dos muestras aleatorias independientes provienen de la misma población, usando el estadístico de Wilcoxon, es una propuesta no paramétrica basada en los rangos de las observaciones originales ${ }^{27-28}$. Los análisis estadísticos fueron realizados mediante el Software InfoStat $2019^{29}$.

\section{Resultados}

La muestra estuvo integrada por un $49 \%$ de pacientes del género femenino y un 51\% del masculino. La Prueba de Concordancia arrojó un valor del estadístico Chi-Cuadrado $\quad 2=0,04$, con un $p$-valor $=0,8415$, lo que indica que no existen diferencias de proporción entre géneros en la composición de la muestra.

Las edades de los pacientes que conformaron la muestra estuvieron comprendidas entre 18 y 73 años, con un promedio de 36 . El 53\% correspondió al grupo etario denominado adultos jóvenes (entre 18 y 35 años), mientras que el $47 \%$ restante se ubicó en el rango de adultos mayores (de 36 años o más).

En la Figura 1, se presentan Gráficos de Caja con la distribución de las edades en ambos géneros, de los que se desprende que no existen grandes diferencias en las edades de los integrantes de la muestra en ambos géneros. Si bien los hombres parecen tener edades más concentradas en valores un poco inferiores, también presentan mayor variabilidad, con valores superiores más extremos, lo que atenúa las diferencias.

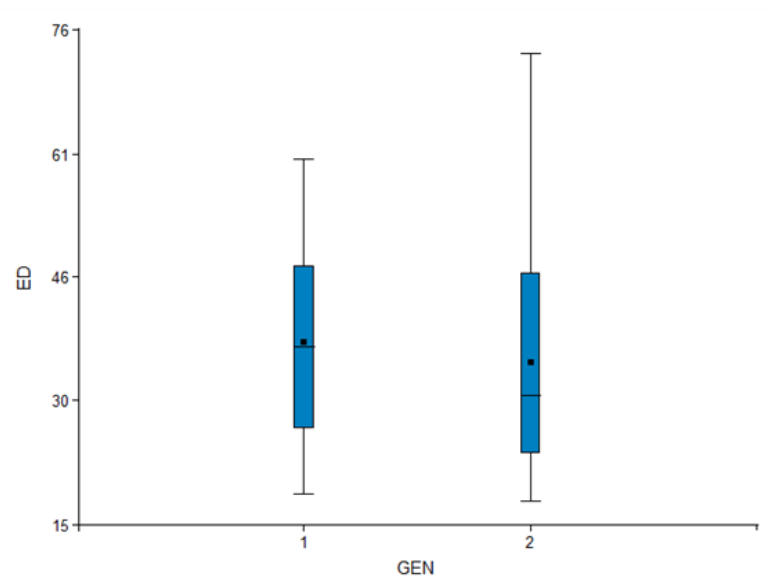

Figura 1. Gráficos de Caja de la edad (ED) por género (GEN), en una muestra de 100 pacientes.

El 59\% de los pacientes que integraron la muestra presentó al menos un signo o síntoma disfuncional de la ATM y pasaron a formar parte del grupo DTM, el $41 \%$ no presentó ningún síntoma o signo de disfunción temporomandibular y constituyeron el grupo control. 


\section{Niveles de ansiedad}

Entre los pacientes de la muestra, al estudiar la ansiedad rasgo se encontró una prevalencia del $34 \%$ en el nivel bajo, un $35 \%$ en el medio y un $31 \%$ en el alto. La prueba de Concordancia arrojó un valor de $\chi^{2}=0,26$ con un $p$-valor $=0,8781$ lo que lleva a aceptar la hipótesis de que no existen diferencias entre las frecuencias con que se presentaron en la muestra los diferentes niveles de ansiedad rasgo. Esto implica decir que los tres valores de la escala de ansiedad rasgo se presentaron en la misma proporción.

Con relación a la ansiedad estado, se pudo determinar un $25 \%$ en nivel bajo, un $58 \%$ medio y un $17 \%$ alto. La prueba de concordancia arrojó un valor de $\chi^{2}=28,34$ con un $p$-valor $<0,0001$, lo que lleva a rechazar la hipótesis de igualdad en las frecuencias con que se presentaron los diferentes niveles y confirmar que, la mayoría de los pacientes presentaron un nivel medio de ansiedad estado, seguido por el nivel bajo y en menor proporción el nivel alto. Con esto se demuestra que, contrariamente a lo que sucede con la ansiedad rasgo, los diferentes niveles de la ansiedad estado no se presentaron en la misma proporción.

Las prevalencias mencionadas se encuentran representadas mediante un Gráfico de Barras en la Figura 2.
Estas variaciones de la ansiedad estado comparadas con un comportamiento más estable de la ansiedad rasgo pueden interpretarse si se tiene en cuenta que la ansiedad-rasgo hace referencia a la ansiedad como tendencia relativamente constante de la personalidad, se refiere a las diferencias individuales, relativamente estables, en la propensión a la ansiedad, es decir, a las diferencias entre las personas en la tendencia a responder a situaciones percibidas como amenazantes, con elevaciones en la intensidad de la ansiedad-estado. Los sujetos con alto grado de ansiedad-rasgo perciben un mayor rango de situaciones como amenazantes y están más predispuestos a sufrir ansiedad-estado de forma más frecuente o con mayor intensidad, pero por otra parte los niveles altos de ansiedad-estado son apreciados como intensamente molestos; por tanto, si una persona no puede evitar el estrés que lo causa, pondrá en marcha las habilidades de afrontamiento necesarias para enfrentarse a la situación amenazante e iniciar un proceso defensivo para reducir el estado emocional irritante $y$, en la medida en que los mecanismos de defensa tengan éxito, las circunstancias se verán menos amenazantes y se producirá una reducción del estado de ansiedad. Asimismo, y de forma general, los sujetos con altos valores de ansiedad-rasgo perciben las situaciones y contextos evaluativos como más amenazantes que aquellos otros que presentan menores niveles de ansiedad-rasgo ${ }^{15-16}$.

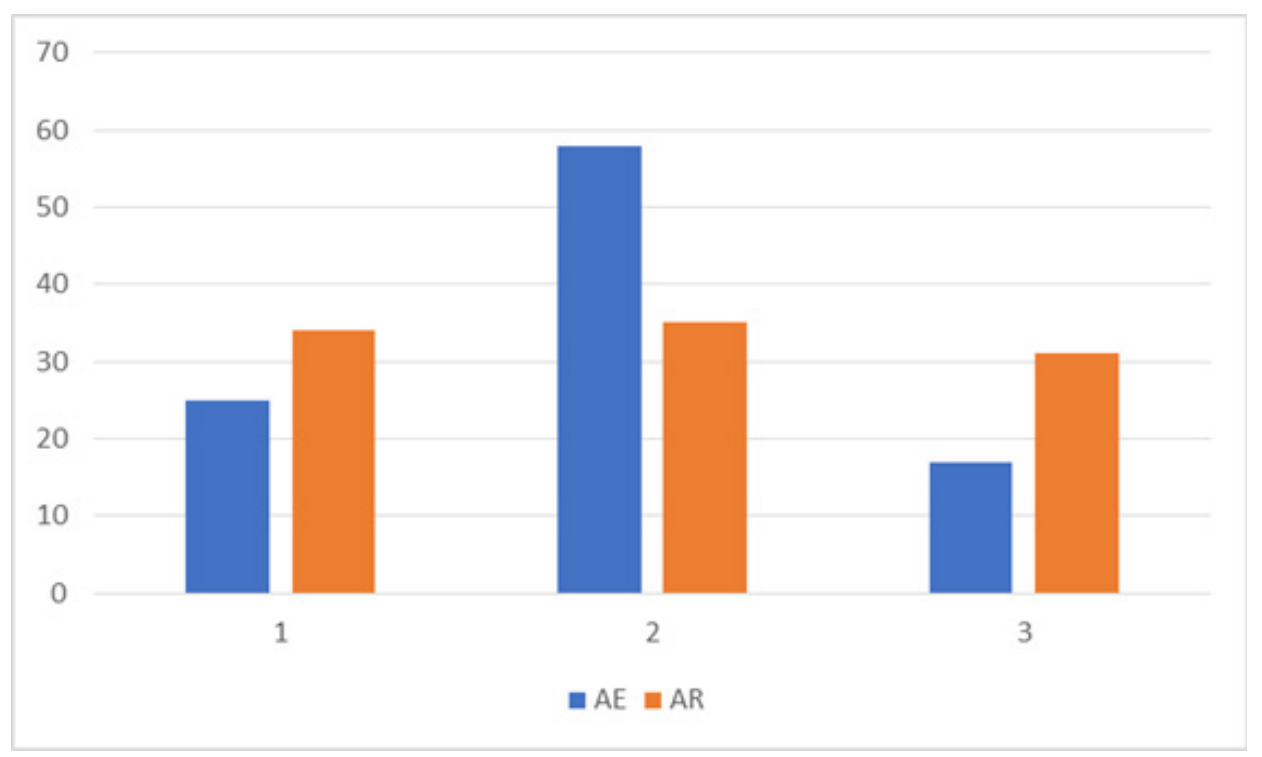

Figura 2. Gráfico de Barras de la prevalencia de los diferentes niveles de ansiedad estado (AE) y ansiedad rasgo (AR), niveles (1) bajo, (2) medio y (3) alto, en una muestra de 100 pacientes. 


\section{Ansiedad rasgo y estado en los diferentes grupos}

En la Tabla I, se presentan las frecuencias de los diferentes niveles de ansiedad rasgo y estado en el grupo Control y DTM, como así también los valores del estadístico $\chi^{2}$ para la hipótesis de independencia de ambas variables y su correspondiente probabilidad.

En el grupo Control, los valores de $\chi^{2}$ y de sus probabilidades en la Prueba de Independencia conducen a afirmar que hay independencia entre las frecuencias de los diferentes valores de ansiedad rasgo y estado. Esto significa que, en el grupo control, las frecuencias con que se presentan los diferentes valores de ansiedad rasgo son independientes de las frecuencias con que se presentan los diferentes valores de ansiedad estado y viceversa.

En el grupo DTM se encontró significancia en la prueba de Independencia, lo que indica que las frecuencias con que se presentan los valores de los diferentes niveles de una variable son diferentes para los distintos niveles de la otra variable. Se puede ver que en el nivel bajo de AR prevalecen los valores bajos de $A E$ y no se observa ningún paciente con nivel alto de $A E$; en el nivel medio de $A R$ se encuentra una distribución uniforme de valores de AE; mientras que en el nivel alto de AR prevalecen los niveles medio $y$ alto de $A E$.
Los pacientes con signos y síntomas de disfunción temporomandibular presentan niveles de ansiedad rasgo y ansiedad estado superiores a los pacientes del grupo control. Entre los pacientes del grupo control, el $37 \%$ presentó ansiedad estado baja, el $61 \%$ media y el $2 \%$ alta, mientras que los pacientes que presentaron algún signo o síntoma de disfunción temporomandibular, se evidenció ansiedad en estado baja en el $17 \%$, mientras que el $56 \%$ presentó ansiedad estado media y el $27 \%$ alta .

Esta diferencia en las respuestas de ambos grupos podría indicar que la presencia de la DTM modifica la relación entre la ansiedad rasgo y la ansiedad estado en los pacientes, idea que se ve reforzada por lo establecido por Spielberger $(1966)^{14}$ que la interacción entre ambos tipos de ansiedad explica por qué la ansiedad-estado puede variar, tanto entre individuos (la característica de ansiedad puede ser diferente ante la misma situación), como individualmente (la misma persona experimenta ansiedad en una situación, pero no en otra).

\section{Grupos etarios}

En la Tabla II, se presentan las frecuencias de los diferentes niveles de ansiedad rasgo y estado en el grupo de adultos jóvenes, como así también los valores del estadístico $\chi^{2}$ para la hipótesis de independencia de ambas variables y su correspondiente probabilidad.

Tabla I. Tabla de Contingencia de las variables ansiedad rasgo y ansiedad estado en los grupos Control y DTM, valores del estadístico estadístico $\chi^{2}$ y su correspondiente probabilidad.

\begin{tabular}{|c|c|c|c|c|c|c|c|}
\hline \multicolumn{8}{|c|}{ ANSIEDAD ESTADO } \\
\hline GRUPO & ANSIEDAD RASGO & 1 & 2 & 3 & TOTAL & $\chi^{2}$ & p-valor \\
\hline \multirow{4}{*}{ Control } & 1 & 11 & 16 & 0 & 27 & \multirow{4}{*}{2,34} & \multirow{4}{*}{0,2759} \\
\hline & 2 & 4 & 9 & 1 & 14 & & \\
\hline & 3 & 0 & 0 & 0 & 0 & & \\
\hline & Total & 15 & 25 & 1 & 41 & & \\
\hline \multirow{4}{*}{ DTM } & 1 & 4 & 3 & 0 & 7 & \multirow{4}{*}{11,67} & \multirow{4}{*}{$0,0200\left(^{*}\right)$} \\
\hline & 2 & 4 & 12 & 5 & 21 & & \\
\hline & 3 & 2 & 18 & 11 & 31 & & \\
\hline & Total & 10 & 33 & 16 & 59 & & \\
\hline
\end{tabular}

(*) indica significancia estadística 
Tabla II. Tabla de Contingencia de las variables ansiedad rasgo y ansiedad estado en el grupo de adultos jóvenes y maduros, valores del estadístico $\chi^{2}$ y su correspondiente probabilidad.

\begin{tabular}{|c|c|c|c|c|c|c|c|}
\hline \multicolumn{8}{|c|}{ ANSIEDAD ESTADO } \\
\hline GRUPO & ANSIEDAD RASGO & 1 & 2 & 3 & TOTAL & $\chi^{2}$ & p-valor \\
\hline \multirow{4}{*}{$\begin{array}{l}\text { Adultos } \\
\text { jóvenes }\end{array}$} & 1 & 10 & 11 & 0 & 21 & \multirow{4}{*}{17,06} & \multirow{4}{*}{$0,0019\left(^{*}\right)$} \\
\hline & 2 & 6 & 13 & 3 & 22 & & \\
\hline & 3 & 0 & 6 & 4 & 10 & & \\
\hline & Total & 16 & 30 & 7 & 53 & & \\
\hline \multirow{4}{*}{$\begin{array}{l}\text { Adultos } \\
\text { mayores }\end{array}$} & 1 & 5 & 8 & 0 & 13 & \multirow{4}{*}{10,12} & \multirow{4}{*}{$0,0385\left(^{*}\right)$} \\
\hline & 2 & 2 & 8 & 3 & 13 & & \\
\hline & 3 & 2 & 12 & 7 & 21 & & \\
\hline & Total & 9 & 28 & 10 & 47 & & \\
\hline
\end{tabular}

$(*)$ indica significancia estadística

En el grupo de adultos jóvenes se encontró significancia en la prueba de Independencia, lo que indica que las frecuencias con que se presentan los valores de los diferentes niveles de una variable son diferentes para los distintos niveles de la otra variable. Se puede ver que en el nivel bajo de AR prevalece el nivel bajo de AE seguido del nivel medio, no se observaron valores altos de $\mathrm{AE}$; en el nivel medio de AR, se observa una distribución casi uniforme de valores de los tres niveles de AE; mientras que en el nivel alto de $A R$ no se encontró ningún paciente con valor bajo de $\mathrm{AE}$ y prevalece el nivel alto de AE.

En el grupo de adultos maduros se encontró significancia en la prueba de Independencia, lo que indica que las frecuencias con que se presentan los valores de los diferentes niveles de una variable son diferentes para los distintos niveles de la otra variable. Se puede ver que en el nivel bajo de AR prevalece el nivel bajo de $A E$ y no se encontró ningún paciente con nivel alto de $A E$; en el nivel medio de $A R$ se observa una distribución uniforme de valores de $A E ; y$ en el nivel alto de AR prevalece el nivel alto de $A E$, seguido del nivel medio.

\section{Discusión}

Como enuncia esta investigación los tres valores de la escala de ansiedad rasgo se presentaron en la misma proporción, siendo que estos valores difieren de los encontrados por Corsini et al. ${ }^{30}$, en una mues- tra de 269 alumnos de Odontología en la Universidad de la Frontera, en Temuco, Chile donde el objetivo fue determinar la frecuencia de los niveles de ansiedad: rasgo y estado en los alumnos de Odontología, el estudio fue de corte transversal basado en una cohorte estratificada por avance curricular, edad y sexo, evaluada con el cuestionario IDARE (Inventario de Ansiedad Rasgo Estado), en el encontraron, en relación con los niveles de ansiedad rasgo, que el $4,1 \%$ fue bajo, $38,5 \%$ medio y el $57,4 \%$ alto.

En este estudio los diferentes niveles de ansiedad estado no se presentaron en la misma proporción, la mayoría de los pacientes presentaron un nivel medio de ansiedad estado, seguido por el nivel bajo y en menor proporción el nivel alto, valores que se aproximan a los encontrados por Corsini et al. ${ }^{30}$, en su estudio sobre estudiantes de Odontología, en los que, en relación con los niveles de ansiedad estado, el $10,7 \%$ de los estudiantes tuvo un nivel bajo, el $53,7 \%$ medio y el $35,6 \%$ alto.

Por otra parte, los valores de ansiedad rasgo y ansiedad estado encontrados en este trabajo indican que todos los pacientes que participaron de la muestra presentaron algún nivel de ansiedad, esto podría deberse al estrés que significa la consulta odontológica, dado que muy superiores son los descriptos por un estudio del Observatorio Nacional De Salud Mental de Colombia realizado en el año 2017, en el cual se aplicó el cuestionario de síntomas SRQ 
(Self-Reporting Questionnaire) para adolescentes y adultos, que evalúa la presencia de síntomas de ansiedad, depresión, psicosis y epilepsia, en adultos de 18 a 44 años. En el que encontraron un 9,6\% de la muestra con síntomas sugestivos de algún trastorno mental, el 52,9\% con uno o más síntomas de ansiedad y el 80,2\% manifiesta de 1 a 3 síntomas depresivos, lo que consideran como evidencia de altos índices de ansiedad presentes en la población adulta en Colombia ${ }^{31}$.

Se puede evidenciar como la ansiedad estado, o sea, la condición o estado emocional de los pacientes, muestra una asociación altamente significativa con los TTM. Trabajos realizados por Domínguez Torres $^{32}$, arrojaron como resultado mayores números en el nivel medio de ansiedad estado (27 de 50), en concordancia con este estudio, al igual que Castillo Hernández ${ }^{1}$, quien obtuvo como resultado mayores números del nivel medio de ansiedad estado ( 26 de $50)$.

Se encontró significancia en la prueba de Independencia, lo que indica que las frecuencias con que se presentan los valores de los diferentes niveles de una variable son diferentes para los distintos niveles de la otra variable. Estos resultados difieren de los hallados por Corsini et al. ${ }^{30}$, quienes encontraron que la edad, presentó asociación significativa y correlación baja con la ansiedad estado, mientras que dicha asociación no fue significativa para la ansiedad rasgo.

\section{Conclusiones}

Todos los pacientes que participaron de la muestra presentaron algún nivel de ansiedad, medida según el Inventario IDARE.

En general se observaron similares prevalencias para los tres niveles ansiedad rasgo, para la ansiedad estado se encontró mayor prevalencia del nivel medio.

En los pacientes con signos y síntomas de disfunción temporomandibular los niveles de ansiedad rasgo y ansiedad estado fueron superiores a los pacientes del grupo control.
En los dos grupos etarios estudiados, los niveles de ansiedad rasgo y ansiedad estado se encuentran directamente relacionados.

\section{Referencias Bibliográficas}

1. Castillo Hernández R, Reyes Cepeda A, González Hernández M, Machado Martínez M. Hábitos parafuncionales y ansiedad versus disfunción temporomandibular. Rev Cubana Ortod. 2001;16(1):14-23.

2. Bomjardim LR, Gaviao MB, Pererira LJ, Castelo PM. Anxiety and depression in adolescents and their relationship with signs 38 and symptoms of temporomandibular disorders. Int J Prosthodont.2005;18(4):347-352.

3. Espinosa-De Santillana I, Reyes-García M, Vaillard-Jiménez E, Vargas García H, Reyes-García Y. Relación de desórdenes temporomandibulares-perfil psicológico en estudiantes de Puebla. Revista Odontológica Mexicana. 2006;10(3):115-118.

4. Peñón Vivas P, Vega Rodríguez Y, Sarracent Pérez H, Pérez Torres FE. Movimientos mandibulares en el síndrome de disfunción temporomandibular. Medimay [Internet]. 2014 [citado 1 Jun 2018]; 20(2). Disponible en: http://revcmhabana.sld.cu/index.php/rcmh/article/view/385/pdf. 5. Lemos GA, Silva PL, Paulino MR, Moreira VG, Beltrão RT, Batista AU. Prevalência de disfunção temporomandibular e associação com factores psicológicos em estudantes de Odontologia. Rev Cubana Estomatol [Internet]. 2015 [citado 1 Jun 2018]; 52(4). Disponible en: http://www.revestomatologia.sld.cu/index.php/est/article/view/764.

6. Galeano AM, Hincapié CL, Ríos NR, Correa I, Vinaccia S. Perfil psicológico del paciente con síndrome de disfunción doloroso temporomandibular. Rev CES Odontología. 2003;16(2):37-4.

7. Poveda Roda R, Bagan JV, Díaz Fernández JM, Hernández Bazán S, Jiménez Soriano Y. Review of temporomandibular joint pathology. Part I: classification, epidemiology and risk factors. Med. Oral Patol Oral Cir Bucal. 2007;12(4):292-8. Disponible en: http://www.medicinaoral.com/medoralfree01/v12i4/medoralv12i4p292.pdf 8. Krogh Paulsen WG, Olsson A. Oclusal disharmonies and dysfunction of the stomatognathic system. Dent Clinic North Am. 1966;Nov:627-35.

9. Krogh Paulsen, WG; Olsson, A. Management of the occlusion of the teeth. In Schwartz L. Chaynes CM. Facial Pain and Mandibular Dysfunction. Philadelphia W.B. Saunders Co.;1969.

10. Mongini F. The Stomatognathic System: Function. Dysfunction and Rehabilitation Chicago. Quintessence. 


\section{Ch. 1983; 6 and 12.}

11. Grunert I, Judmaier W. Informe del caso de una paciente con prolapso discal en ambas articulaciones temporomandibulares. Exploración muscular según Krogh - Poulsen. Quintessence. 1993;6(3):162-173.

12. Cia AH. La ansiedad y sus trastornos: Manual diagnóstico y terapéutico. En La ansiedad y sus trastornos: manual diagnóstico y terapéutico. 2007; p. 494-494.

13. Vimpari SS, Knuuttila ML, Sakki TK, Kivela SL. Deppressive symptoms associated with symptoms of the temporomandibular joint pain and dysfunction syndrome. Med. 1995;57(5):439-444. DOI: 10.1097/00006842199509000-00005

14. Spielberger CD. Anxiety and behavior. New York: Academic Press; 1966.

15. Wadey R, Hanton S. Basic psychological skills usage and competitive anxiety responses: perceived underlying mechanisms. Res Q Exerc Sport. 2008;79(3):363-373. DOI: 10.1080/02701367.2008.10599500

16. Mellalieu SD, Hanton S. (Eds.). A competitive anxiety review: Recent directions in sport psychology research. Hauppauge, Nueva York: Nova Science;2009.

17. Mellalieu SD, Neil R, Hanton S, Fletcher D. Competition stress in sport performers: Stressors experienced in the competition environment. J Sports Sci. 2009; 27(7):729-744. DOI: 10.1080/02640410902889834.

18. Hackfort D, Spielberger CD. (Eds.). Anxiety in Sports. An International Perspective. New York,NY:Hemisphere;1989.

19. Observatorio Nacional de Salud Mental. Guía Metodológica para el Observatorio Nacional de Salud Mental. MINSALUD, 2017; 47.

20. Spielberger CD, Díaz-Guerrero R. IDARE. Inventario de Ansiedad: Rasgo-Estado. México: Editorial El Manual Moderno;1975.

21. Arias Galicia LF. La escala de satisfacción marital: análisis de su confiabilidad y validez en una muestra de supervisores mexicanos. Rev Interam Psicol. 2003;37(1):67-92. DOI: 10.30849/rip/ijp.v37i1.809

22. Rojas Carrasco KE. Validación del Inventario de Ansiedad Rasgo-Estado en padres con un hijo en terapia intensiva. Rev. Med Inst Mex Seguro Soc. 2010;48(5):491496. Disponible en: https://www.redalyc.org/ pdf/4577/457745510005.pdf

23. Quispe Calizaya RP. Relación entre niveles de ansiedad y trastornos temporomandibulares en estudiantes de la Escuela Académico Profesional De Odontología de la Universidad Nacional Jorge Basadre Grohmann, Tacna, Perú. Tesis para optar al título de Cirujano Dentista.
2014;133 págs. Disponible en: http://repositorio.unjbg. edu.pe/handle/UNJBG/2021.DOI:10.33326/26644649. 2014.2.2.807

24. Hernández Romero Y, Tápanes Domínguez A, Casanova Díaz C, Cárdenas Peña L, Saavedra de la Cruz A. Niveles de ansiedad y depresión en un grupo de pacientes con trastornos en la articulación temporomandibular / Anxiety and depression levels in a group of patients with temporomandibular articulation Rev. Hosp. Psiquiátrico de la Habana. 2013;10(1):7-11. Disponible en: http:// www.revistahph.sld.cu/hph0113/hph05113.html. DOI: 10.24875/BMHIM.20000002

25. Machado ME, Fasanella MR. Ansiedad y disfunción temporomandibular. Odous Científica. 2009;10(1):10-7.

26. Luna Yañez, C. Estrés y su relación con la disfunción temporomandibular en Trabajadores de empresa privada. Revista Salud, Sexualidad y Sociedad, 2010;2(4):1-3. DOI: 10.1544/rsap.v18n1.47613

27. Balzarini MG, Di Rienzo JA. Introducción a la Bioestadística. Aplicaciones con InfoStat. Argentina: Ed. Brujas; 2011.

28. Díaz-Guerrero R, Spielberger CD. IDARE: Inventario de ansiedad: rasgo-estado. México: El Manual Moderno; 1975.

29. Di Rienzo JA, Casanoves F, Balzarini MG, Gonzalez L, Tablada M, Robledo CW. InfoStat versión 2018. Grupo InfoStat, FCA, Universidad Nacional de Córdoba, Argentina. URL http://www.infostat.com.ar.

30. Corsini GM, Bustos LM, Fuentes JN, Cantín M. Niveles de Ansiedad en la Comunidad Estudiantil Odontológica. Universidad de La Frontera, Temuco - Chile. Int. J Odontostomat. 2012;6(1):51-57. DOI: 10.4067/S0718381X2012000100007

31. Villarreal Sánchez JS, Ortíz Martínez LV, Fandiño Vigoth KV. Prevalencia de ansiedad en población universitaria de la ciudad de Bucaramanga, según el cuestionario de Ansiedad Estado-Rasgo (STAI), 2019. Disponible en: https:// repository.ucc.edu.co/handle/20.500.12494/13825

32. Domínguez Torres L, Guerra Pérez P, Catañeda Molerio R. La disfunción temporomandibular y su relación con la ansiedad y los hábitos parafuncionales. Mediciego [revista en internet]. 2013 [citado 18 febrero 2018]; 19(2) Disponible en: http://www.bvs.sld.cu/revistas/mciego/ vol19_no2_2013/pdf/T2.pdf 